Supporting Information

\title{
Controllable threshold voltage in organic complementary logic circuits with an electron-trapping polymer and a photoactive gate dielectric layer
}

Toan Thanh Dao, ${ }^{\dagger}, *$ Heisuke Sakai,${ }^{\ddagger}$ Hai Thanh Nguyen, ${ }^{\dagger}$ Kei Ohkubo, ${ }^{\S, \#, \|}$ Shunichi Fukuzumi, $\S, \#, \|$ and Hideyuki Murata ${ }^{*}, *$

${ }^{\dagger}$ Faculty of Electrical-Electronic Engineering, University of Transport and Communications, No. 3, Cau Giay Street, Dong Da, Hanoi, Vietnam

Japan Advanced Institute of Science and Technology, 1-1 Asahidai, Nomi, Ishikawa 923-1292, Japan

${ }^{\S}$ Department of Material and Life Science, Graduate School of Engineering, Osaka University, Suita, Osaka 565-0871, Japan

\#Department of Chemistry and Nano Science, Ewha Womans University, Seoul 120-750, Korea. ${ }$ Faculty of Science and Technology, Meijo University, ALCA and SENTAN, Japan Science and Technology Agency (JST), Nagoya, Aichi 468-8502, Japan

\section{Corresponding Authors}

Dr. T. T. Dao (E-mail: daotoan@utc.edu.vn)

Faculty of Electrical-Electronic Engineering, University of Transport and Communications, No. 3, Cau Giay Street, Dong Da, Hanoi, Vietnam.

Prof. H. Murata (E-mail: murata-h@jaist.ac.jp)

Japan Advanced Institute of Science and Technology, 1-1 Asahidai, Nomi, Ishikawa 923-1292, Japan. 
(a)

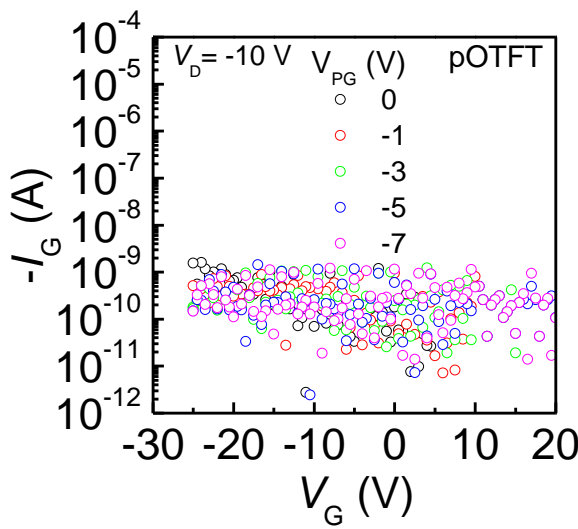

(b)

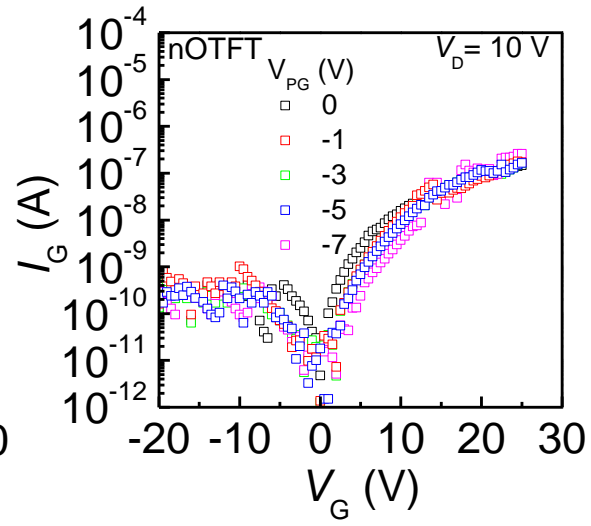

Figure S1. Gate current $\left(I_{\mathrm{G}}\right)$ of (a) pOTFT or (b) nOTFT measured together with transfer characteristic measurements. $V_{\mathrm{D}}$ values were set to be $-10 \mathrm{~V}$ for pOTFT and $10 \mathrm{~V}$ for nOTFT in all measurements.

We have plotted the gate leakage current during the transfer characterizations in Figure S1. For the pOTFT, when $V_{\mathrm{G}}$ was varied from positive to negative, the gate current $\left(I_{\mathrm{G}}\right)$ fluctuated from approximately $10^{-12}$ to $10^{-9} \mathrm{~A}$. Meanwhile, for the nOTFT, with negative values for $V_{\mathrm{G}}$, the $I_{\mathrm{G}}$ of the device was also within the small range, $10^{-12}-10^{-9} \mathrm{~A}$; however, $I_{\mathrm{G}}$ increases from approximately $10^{-12}$ to $10^{-7}$ A with a positive $V_{\mathrm{G}}$. At $V_{\mathrm{PG}}=25 \mathrm{~V}, I_{\mathrm{G}}\left(1.65 \times 10^{-7} \mathrm{~A}\right)$ is approximately two orders of magnitudes lower than $I_{\mathrm{D}}\left(1.51 \times 10^{-5} \mathrm{~A}\right)$. The relatively high leakage current in the nOTFT could be owing to the fact that a part of the photoelectron passes through the $\mathrm{Cytop} / \mathrm{C}_{60} / \mathrm{Cu}$ multi-layer. In our previous work, ${ }^{1}$ through the current density-voltage characteristics measurement of the ITO $(150 \mathrm{~nm}) / \mathrm{C} 60(50 \mathrm{~nm}) / \mathrm{Cytop}(10 \mathrm{~nm}) / \mathrm{C} 60(50 \mathrm{~nm}) / \mathrm{Al}$ $(70 \mathrm{~nm})$, we have determined that the electrons can pass through the 10-nm-thick Cytop layer. For the nOTFT, the energy difference between the LUMO of $\mathrm{C}_{60}(4.5 \mathrm{eV})^{1}$ and the work function of $\mathrm{Cu}(4.6 \mathrm{eV})^{2}$ is considerably small (about $0.1 \mathrm{eV}$ ). Thus, it is obvious that a current can flow through the multi-layer, Cytop/C60/Cu, resulting in an increase in the observed gate current in the positive region of $V_{\mathrm{G}}$. However, the gate currents of the pOTFT in the entire $V_{\mathrm{G}}$ range and gate currents of the nOTFT with negative $V_{\mathrm{G}}$ values did not increase because holes cannot pass through the Cytop layer. 
(a)

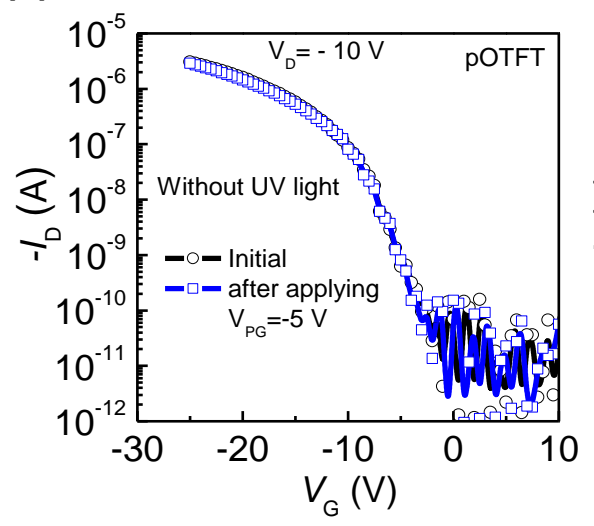

(b)

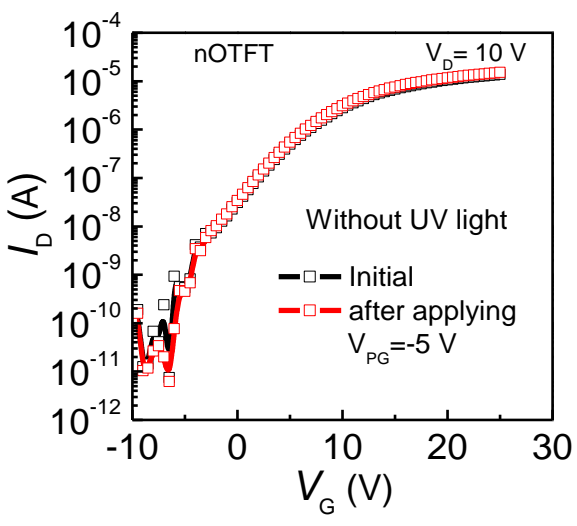

Figure S2. Transfer curves of (a) pOTFT and (b) nOTFT devices measured after applying $V_{\mathrm{PG}}$ of $-5 \mathrm{~V}$ for pOTFT or nOTFT for $1 \mathrm{~s}$ and without UV light irradiation. As can be seen, transfer curves almost did not change in both cases of pOTFT or nOTFT. 
(a)

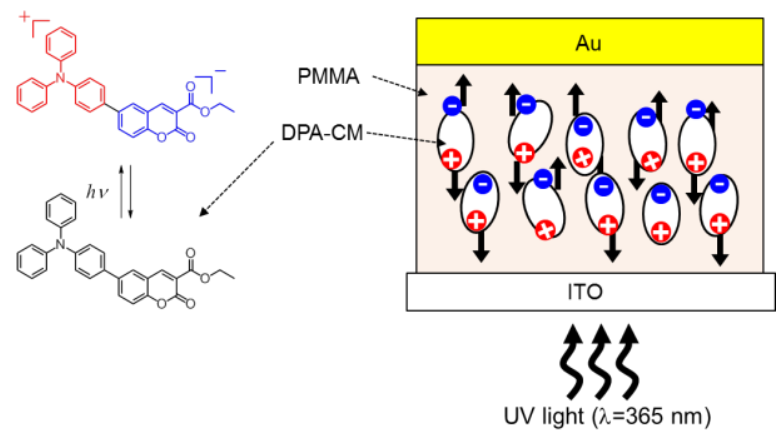

(b)

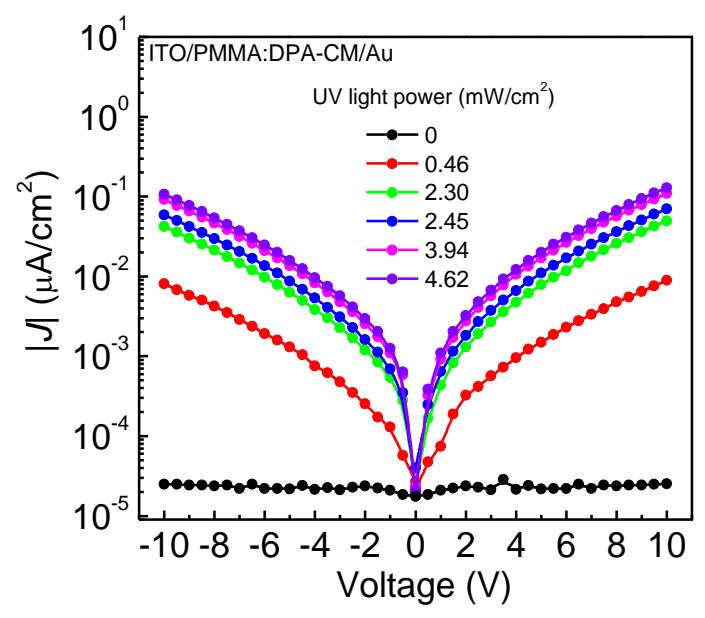

Figure S3. (a) Device structure and (b) current-voltage measurement of the ITO (150 $\mathrm{nm})$ /PMMA:DPA-CM/Au (50 nm) photoactive dielectric film measured under dark and different UV light $(365 \mathrm{~nm})$ power values. 


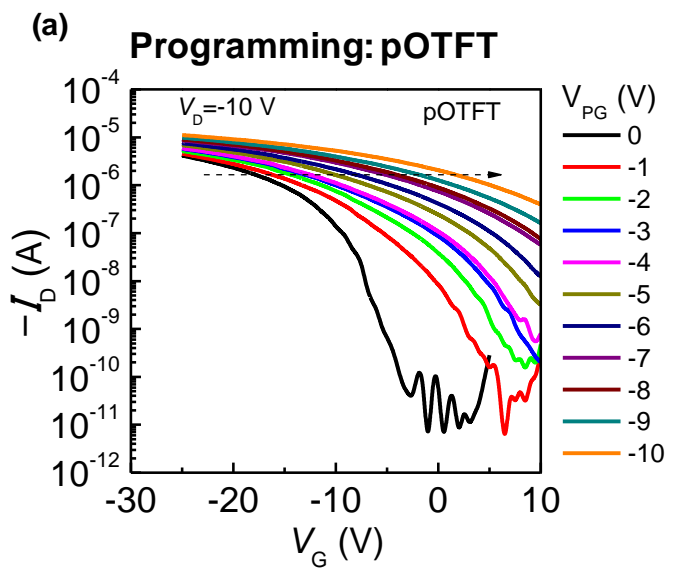

\section{Erasing:pOTFT}

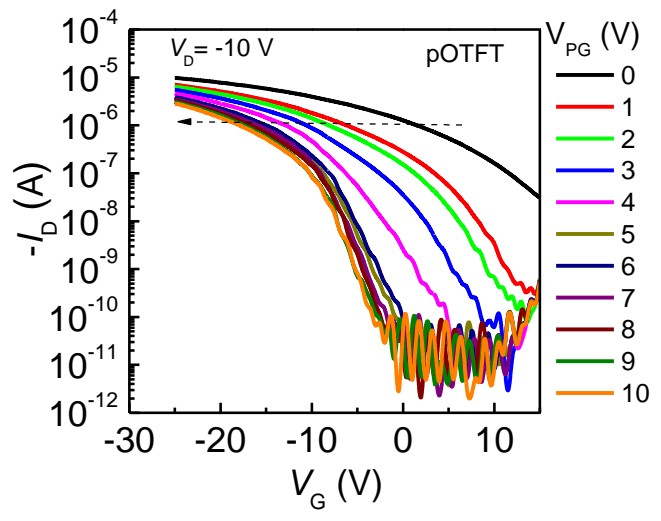

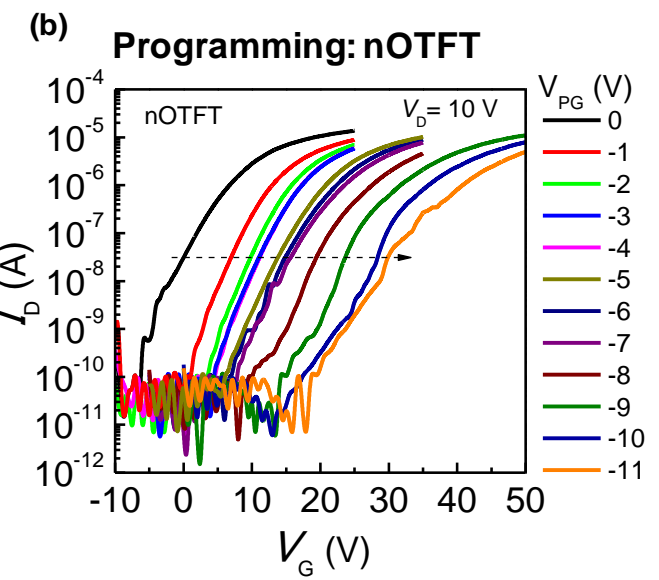

Erasing: nOTFT

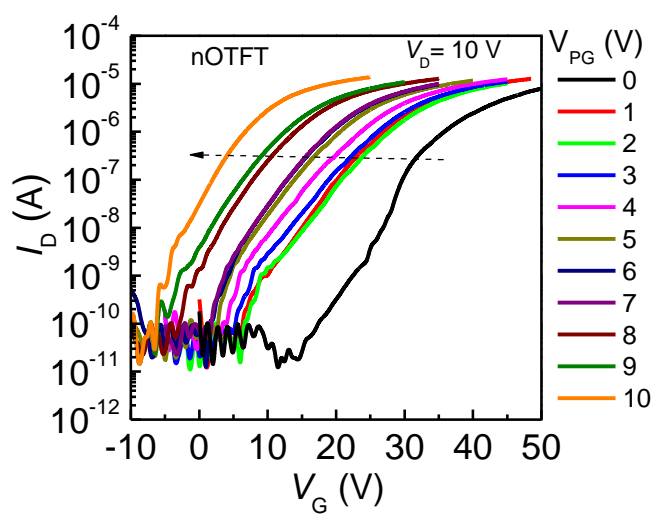

Figure S4. Transfer curves of (a) pOTFT and (b) nOTFT measured after programming (top) and erasing (bottom). Programming or erasing operations were done by applying $V_{\mathrm{PG}}$ for $1 \mathrm{~s}$ under UV light intensity of $3.94 \mathrm{~mW} / \mathrm{cm}^{2}$ while source and drain electrodes were grounded. 
(a)

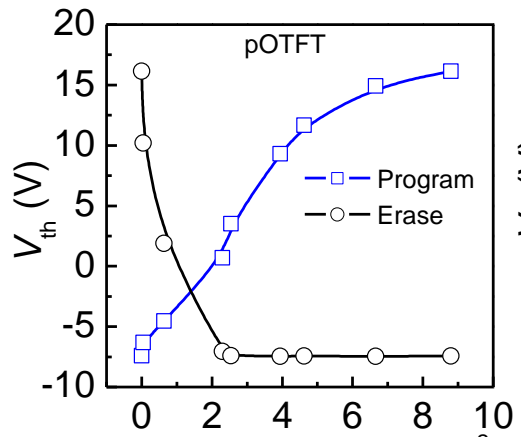

Light intensity $\left(\mathrm{mW} / \mathrm{cm}^{2}\right)$

(c)

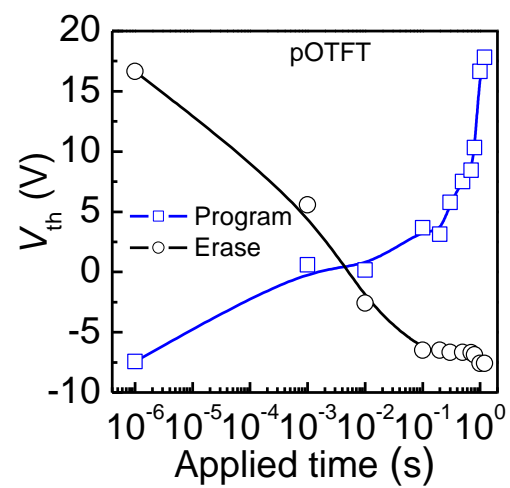

(b)

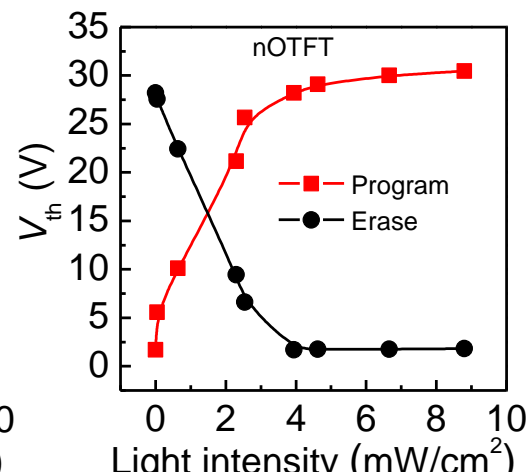

(d)

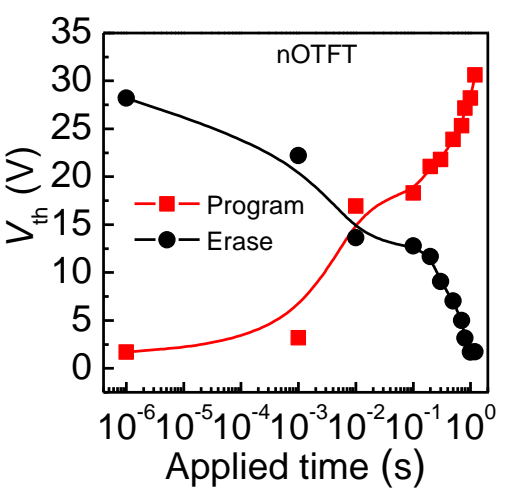

Figure S5. $V_{\text {th }}$ shift of (a) pOTFT or (b) nOTFT as function of UV light intensity with $V_{\text {PG }}$ of $-10 / 10 \mathrm{~V}$ and applied time of $1 \mathrm{~s}$. Change in $V_{\text {th }}$ of (c) pOTFT or (d) nOTFT as a function of applied time with $V_{\mathrm{PG}}$ of $-10 / 10 \mathrm{~V}$ with $\mathrm{UV}$ light intensity of $3.94 \mathrm{~mW} / \mathrm{cm}^{2}$. $V_{\mathrm{DD}}$ values were set to be $-10 \mathrm{~V}$ for pOTFT and $10 \mathrm{~V}$ for nOTFT in all transfer curve measurements.

The reversible shifts in the $V_{\text {th }}$ as a function of the UV light intensity are investigated (Figures $\mathrm{S} 5(\mathrm{a})$ and (b)). The $V_{\text {th }}$ was reversely controlled from -7.4 to $+16.7 \mathrm{~V}$ for the pOTFTs, and from +1.7 to $+30.6 \mathrm{~V}$ for the nOTFTs, respectively. At the programming state of pOTFTs, $V_{\text {th }}$ gradually increased with the increase in the UV light intensity and nearly unchanged at UV light intensity higher than $6.6 \mathrm{~mW} / \mathrm{cm}^{2}$. Meanwhile, at the erasing state, the decrease in $V_{\text {th }}$ saturated at the UV light intensities higher than $2.30 \mathrm{~mW} / \mathrm{cm}^{2}$. On the other hand, at the programming or erasing state of nOTFTs, the changes in $V_{\text {th }}$ almost saturated at the UV light intensity of 3.94 $\mathrm{mW} / \mathrm{cm}^{2}$. Based on $V_{\text {th }}$ changes versus UV light intensities in both circuit devices, we chosen a UV light intensity of $3.94 \mathrm{~mW} / \mathrm{cm}^{2}$ for programming and erasing the organic complementary circuit. 
The reversible shifts in the $V_{\text {th }}$ as functions of the applied time for $V_{\mathrm{PG}}$ are investigated (Figures S5(c) and (d)). At the programming/erasing state of both pOTFTs and nOTFTs, $V_{\text {th }}$ gradually shifted with the increase in the applied time of $V_{\mathrm{PG}}$. In both pOTFT and nOTFT, the large $\mathrm{V}_{\text {th }}$ shift of c.a. $30 \mathrm{~V}$ for the $1 \mathrm{~s}$ duration of $V_{\mathrm{PG}}$ application is enough large to obtain the programming/erasing state. The applied time is similar to that in previous work. ${ }^{3}$ 
(a)

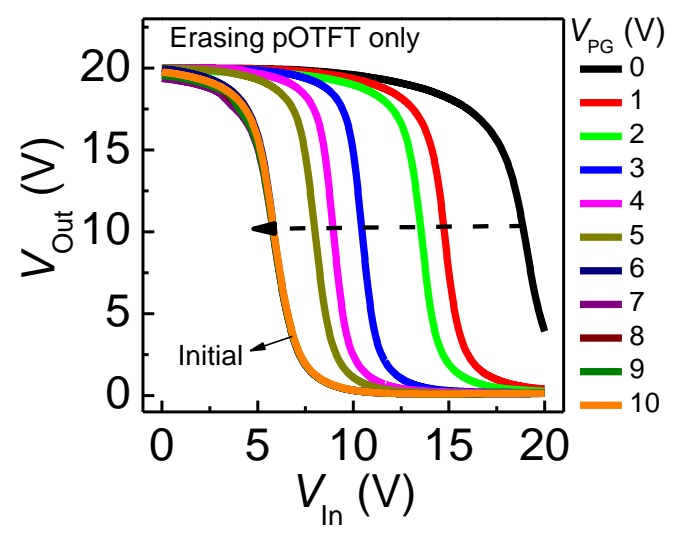

(c)

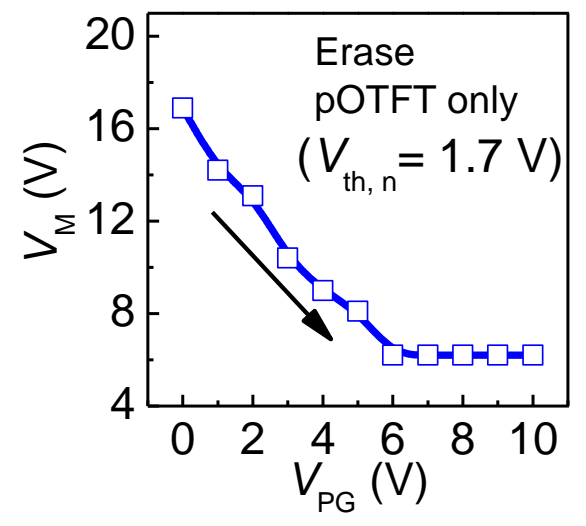

(b)

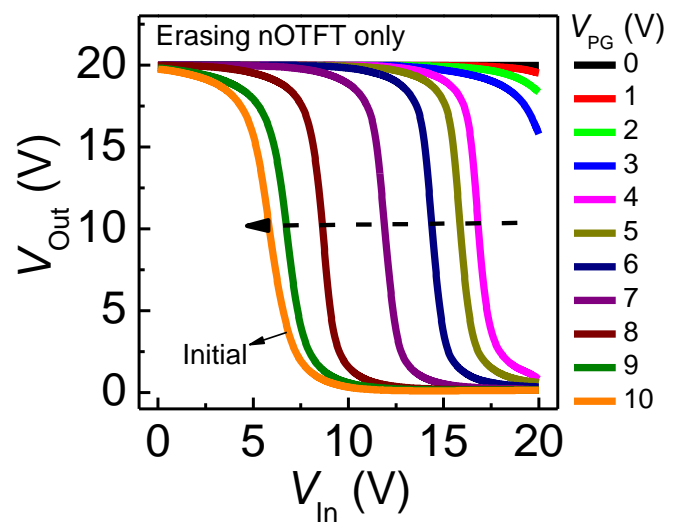

(d)

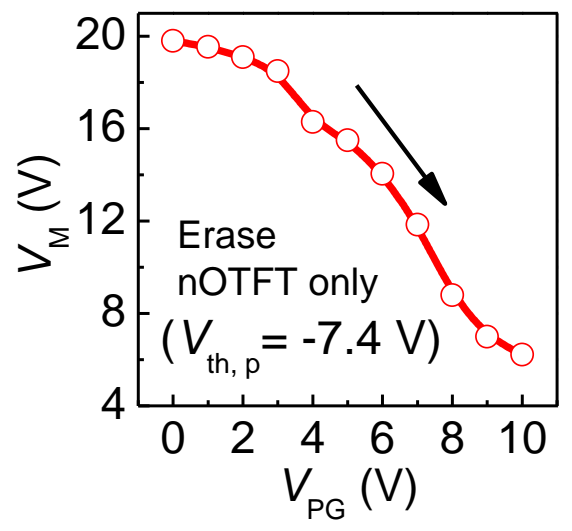

Figure S6. VTC behaviors of circuit after erasing (a) pOTFT and (b) nOTFT. Changes of $V_{M}$ of circuit as function of $V_{\mathrm{PG}}$ voltage for (c) pOFET and (d) nOFET. For pOTFT erasing process, the $V T C$ almost returns to the initial position at a $V_{\mathrm{PG}}$ of $6 \mathrm{~V}$. While, in the case of nOTFT erasing, a $V_{\mathrm{PG}}$ of $10 \mathrm{~V}$ is needed to make the $V T C$ come back the initial value. 
(a)

Programming pOTFT

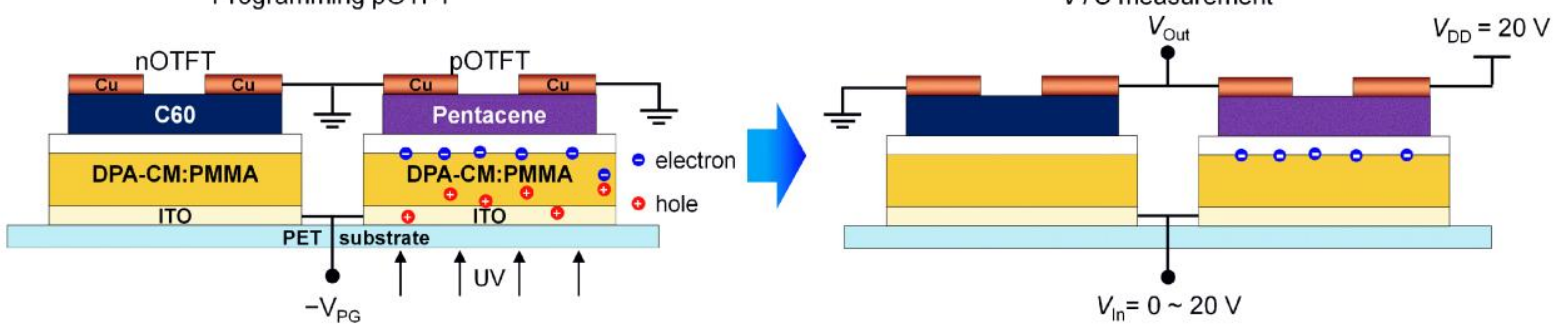

(b)

Programming nOTFT
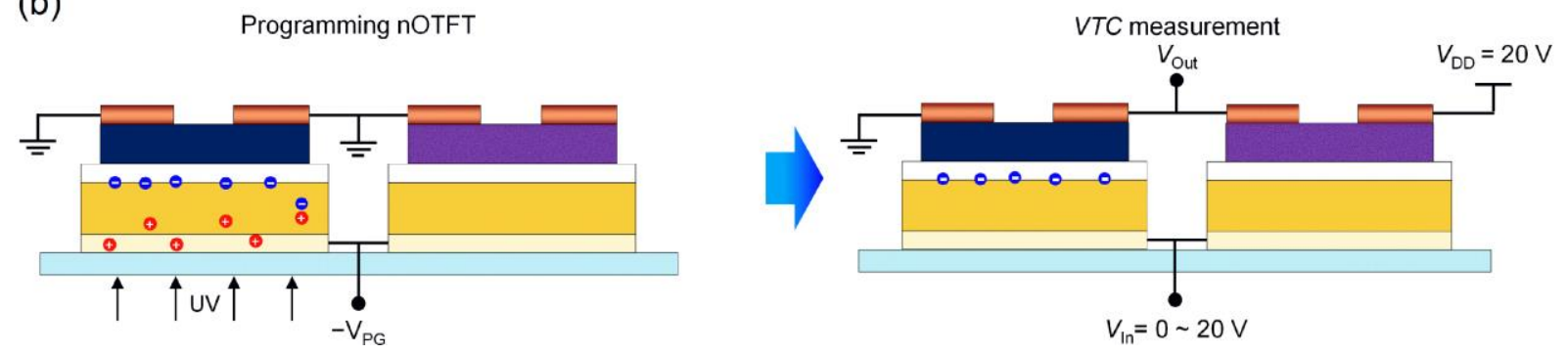

Figure S7. Illustrations of $V T C$ measurements at $V_{\mathrm{DD}}=20 \mathrm{~V}$ after programming (a) pOTFT or (b) nOTFT. To measure effects of pOTFT programming, nOTFT was set at the initial state and pOTFT was programmed, and then VTC measurement was performed under dark. To measure effects of nOTFT programming, pOTFT was set at the initial state and nOTFT was programmed, and then VTC measurement was done under dark. 


\section{References}

(1) Dao, T. T.; Matsushima, T.; Murata, H. Organic Nonvolatile Memory Transistors Based on Fullerene and an Electron-Trapping Polymer. Org. Electron. 2012, 13, 2709-2715.

(2) Su, Y.; Xie, W.; Xu, J. Facile Modification of Cu Source-drain (S/D) Electrodes for HighPerformance, Low-Voltage n-channel Organic Thin Film Transistors (OTFTs) Based on C60. Org. Electron. 2014, 15, 3259-3267.

(3) Yokota, T.; Nakagawa, T.; Sekitani, T.; Noguchi, Y.; Fukuda, K.; Zschieschang, U.; Klauk, H.; Takeuchi, K.; Takamiya, M.; Sakurai, T.; Someya, T. Control of Threshold Voltage in Lowvoltage Organic Complementary Inverter Circuits with Floating Gate Structures. Appl. Phys. Lett. 2011, 98, 193302. 